\title{
Studying the mechanisms of the Somatic Marker Hypothesis in Spiking Neural Net- works (SNN)
}

\author{
Alejandro Jiménez-Rodríguez, Luis F. Castillo ${ }^{\mathrm{b}, \mathrm{c}}$, Manuel Glez \\ Bedia $^{\mathrm{d}}$ \\ ${ }^{a}$ Laboratorio de Neurofisiología, Grupo Neuroaprendizaje, Universidad Autónoma de Manizales \\ ${ }^{\mathrm{b}}$ Grupo Investigación Ing. Del Software, Universidad Autónoma de Manizales \\ ${ }^{\mathrm{b}}$ Grupo Investigación GITIR, Universidad de Caldas \\ ${ }^{\mathrm{b}}$ Computer Science Department, Universidad de Zaragoza (Spain)
}

KEYWORD

Somatic Marker Hypothesis Spiking Neural Networks Decision Making

Emotions

\section{Introduction}

In 1848 , an incident with a patient, whose name was Phineas Gage, revealed a previously unsuspected aspect of emotions and its relation with the decision making [ MACMILLIAN, M. 2009 ]. In the accident, his prefrontal cortex resulted seriously comprised [DAMASIO, H. et al. 1994] and, as a consequence, many of his executive functions was impaired to the extent that his relatives described him as: "no longer Gage", when talking about his sharp personality changes, which included abnormalities in emotion and feeling, incapacity of successful action planning and a marked dysfunction in his decision making capabilities [DAMASIO, A. 1994].

Throughout the years, many other patients had shown similar profiles after a trauma in the prefrontal cortex, having a normal intellect but emotion related abnormalities and an impaired decision making; also, the case of Phineas Gage have been re-examined and even misused in a variety of studies from the anatomical and cognitive point of view [MACMILLIAN, $\mathrm{M}$. 2009]. In particular, the Neurologist Antonio Damasio, used that evidence to suggest the Somatic Marker Hypothesis, in which, the process of decision making is assisted by a somatic cue or signal that is a reenaction of the consequences, appetitive or aversive, of the competing options based on past experiences of similar instances of the problem [DAMASIO, A. 1991]. To support the hypothesis, Damasio and collaborators designed tests to elicit an emotional response in the subjects and measure the somatic response by means of physiological variables like the Galvanic Skin Response (GSR). Other more elaborated test was called the Iowa Gabling Task, in which the subject had to play a game consisting in making a sequence of choices of cards from a deck with corresponding monetary rewards or punishments that lead to long-term winning and losing strategies. The pa- 
tients with damage in the prefrontal cortex, in particular, the VentroMedial Prefrontal Cortex, appeared to be unable to learn from previous mistakes choosing most of the time the wrong strategies [BECHARA, A. et. al. 2005].

The influence of emotions in decision making has become an important topic of research nowadays and new results are shedding light about its structural and functional substrates in the human brain, some modern accounts of the conceptual aspects related to emotions, can be found in [LEDOUX, J. 2000] and [ROLLS, E. et. al. 2008]. This new focus on emotions is having direct applications in areas that range from the economical science, where an entire new field of research known as Neuroeconomics [GLIMCHER, P. et. al. 2009] has emerged and fundamental concepts like the expected utility are being redefined; to robotics and Human Machine Interaction, where the realization of emotions as a driver in the natural interaction with the world, is triggering new developments to emulate those capabilities in artificial agents, giving rise to fields like Affective Computing. Regarding to this, the computational and theoretical details of the neural implementation of such emotional decision making are important and widely studied in modern affective neuroscience. In particular, the construction of models has served as a guide and a proof of concept of the many proposed mechanisms in all levels of complexity.

The use of Spiking Neural Networks (SNN) to simulate neuronal phenomena is being increasingly accepted in neuroscience, as an efficient alternative to construct biologically plausible models of neural networks. In this king of models, each node is a dynamical representation of a simplified neuron that fire according to some changes in its state triggered by the synaptic interaction with other neurons [FELLOUS, J. et al. 2005]. There is some previous work in the modeling of emotional circuits using SNN. In particular, the GAGE and ANDREA models, proposed in [THAGARD, P. 2008] and [LITT, A. et. al. 2008], were used to support hypothesis about the neural structures and the integration of emotional, contextual and rational information in the process of decision making. In this work we use SNN to study the proposed mechanisms of the SMH in the light of the current work in Neuroeconomics about the representations of Objective and Emotional Value, using a more detailed model of the pyramidal neurons of the cortex where the decision is make.

\section{Material and methods}

The NEURON simulation environment was used to run the simulations with an adaptive step integration method implemented through CVODE, using an Event Based Simulation Framework. The general methodology to work with large networks as needed in this work is the following:

a. The general neuron model is specified along with its specific biophysical variables.

b. A prototype network is constructed using the NEURON's GUI

c. The Network is modified and make massive using the Hoc Programming Language

\subsection{Neuron Models}

There are two main types of neurons that were used in the simulations.

The first are Integrate and Fire (IF) neurons (Artificial Neurons) which are punctual units that can be seen as leaky integrators. This kind of neurons can fire spontaneously and, therefore, be used as input to the recurrent and associative networks. Each IF neuron is specified with a particular firing rate, an average number of spikes and certain level of noise, if required. A combination of IF neurons makes up the input stimuli, coded in its different firing rates.

The second kind is Biophysical Neurons, they have a spatial extent, with soma and neurites in which the voltage changes and the ionic currents are implemented using the Hodgkin-Huxley dynamics and the spatio-temporal behavior follows the cable equation, see, for example [DAYAN, P. et al. 2001]. The biophysical parameters of the pyramidal neurons of the model are shown in the table 1 . The spatial shape of the neuron is defined in the GUI as shown in Figure 1.

This type of neurons imposes a heavy task on the integrator and, in a massive model, has a high computational cost. 


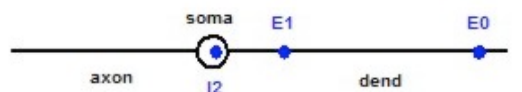

Fig. 1. Pyramidal Neuron Model as specified in the NEURON Simulator GUI

\begin{tabular}{ll}
\hline Variable & Value \\
\hline Diameter (soma) & $24 \mu \mathrm{m}$ \\
Length (Dendritic Tree) & $1000 \mu \mathrm{m}$ \\
Length (Axon) & $7000 \mu \mathrm{m}$ \\
Diameter (Dendrite) & $2.5 \mu \mathrm{m}$ \\
Diameter (Axon) & $1 \mu \mathrm{m}$ \\
$g_{N A}$ (Soma y Axón) & 0.12 \\
$g_{K}$ (Soma y Axón) & 0.036 \\
$g_{\text {Leakage }}$ (Soma y Axón) & 0.0003 \\
Cytoplasmatic Resistance & $150 \Omega \mathrm{m}$ \\
Dendritic Membrane Conductance & $1 / 15000 \mathrm{~S} / \mathrm{cm}^{2}$ \\
Dendrite equilibrium potential & $-63 \mathrm{mV}$
\end{tabular}

Table. 1. Morphological and physiological properties of the prototype pyramidal neuron

2.2. Neural Networks and Synaptic Mechanisms

All the neurons are stored in a vector and its events are managed by a NetCon Object that dispatch the spikes to all the units connected to the source neuron. In the IF neurons, the synapses are implemented as logical events called NET_RECEIVE. In the Biophysical Neurons, the synapses are implemented as Point Processes (POINT_PROCESS) which have local effects in the membrane conductance of the postsynaptic neuron. In figure 1 is shown how are they specified in the neuron GUI, it can be seen that the kind of synapse is specified in the design, generally, the inhibitory synapses are put in the soma [CARNEVALE, N, 2005].

To implement the plasticity in associative networks it is necessary to define some kind of Long/Short Term Potentiation Mechanism, dependent of the action potential of the pre-synaptic neuron. Although the exact molecular mechanisms related with memory are still a matter of research, it is possible to obtain an analog to the Hebb by means of the Synaptic Time Dependent Plasticity (STDP), in which the weight of the syn- apse is strengthened if there are pre and post synaptic spikes concurrently on a certain time window. To implement this on NEURON, a POINT_PROCESS is created on a Biophysical Neurons in such a way that its conductance $g$ increase according to the equations 1 and 2, such mechanism is shown in figure 2 as shown in NEURON's GUI.

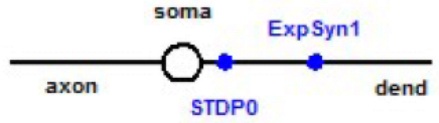

Fig. 2. Neuron Model with Synaptic Time Dependent Plasticity (STDP)

$$
\begin{gathered}
g=g_{0}+w(1+A) \\
A=A_{0}+\alpha \exp \left(\left(t_{\text {pre }}-t_{\text {pos }}\right) / \tau\right)
\end{gathered}
$$

Equation 1 expresses the change in conductance of the cellular membrane in terms of some increment $\mathrm{A}$ multiplied by a weight $w$, which, contrary to the conventional Hebbian rule, does not get affected by the learning, is just a parameter of NET_RECEIVE in NEURON. The synapse get affected (Potentiated or Depressed) by the more biologically plausible mechanism specified in equation 2 whose change depends proportionately on the synchrony of pre and postsynaptic events.

\section{Theory}

\subsection{Proposed Model}

The model in figure 3 is synthesized according to the main anatomical an physiological information available in the literature related to the emotions and its influence in decision making [FELLOUS, J. 2007]. The focus in this work is to explore in-silico implementations of the kind of networks present in the structures marked with a red ellipse. However, let us give a short account to the big picture as shown in the conceptual model.

First, appropriate representations of the value [PLATT, M. et. al. 2009] are associated or recalled given a specific stimulus, in the Lateral Intra Parietal and the OrbitoFrontal Cortexes, regarding what we 
called the Objective Value, associated to the expected utility in terms of rational considerations; and in the Ventro Medial Prefrontal Cortex and the Amygdala, regarding the emotional subjective value ${ }^{1}$. The outputs of such networks is the integrated in an unified Real Subjective Value that will drive the behavior after the integration with contextual information (framing) [LITT, A. et. al. 2008] in the Nucleus Accumbens (Nacc). The kind of response, autonomic and behavioral are instrumented through subcortical mechanisms that go beyond this article. The consequences of the implemented action are then feed again, coded in specific body states, in the main emotional structures, through the Insula and the Anterior Cingulate Cortex (ACC), shaping what is called a Somatic Marker that control the emotional weight associated with that (class of) stimulus.

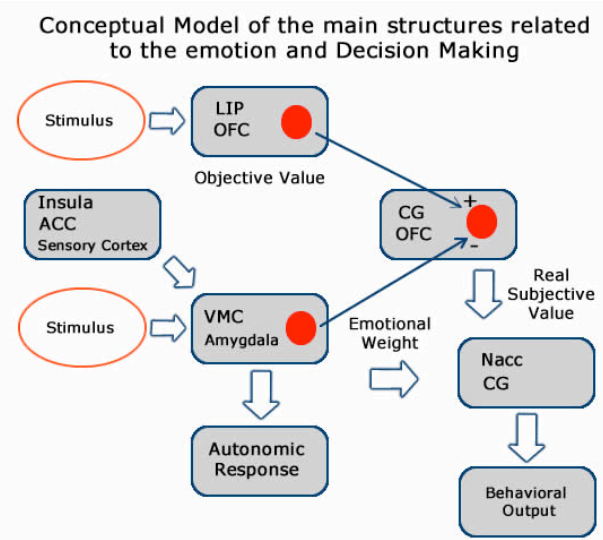

Fig. 3. Conceptual model of the main structures involved in emotions and decision making. ACC: Anterior Cingulate Cortex, VMC: VentroMedial Prefrontal cortex, OFC: Orbito-Frontal Cortex, Nacc: Nucleus Accumbens, CG: Cingulate Cortex. LIP Lateral Intraparietal Cortex.

\section{Results}

\subsection{Associative Networks (Objective/ Subjective Value)}

\footnotetext{
${ }^{1}$ It is unavoidable having uncertainty to some extended in the real circuits involved here, due to the still changing amount of information that exists in the literature.
}

We focus first in the potentiation effect obtained by the implementations of the STDP mechanism in a Pyramidal Neuron. The contrary effect (Depression) in the synapse is not taken into account. As shown in the figure 4, two IF neurons, S22 y S11, are connected to a pyramidal one with Non-STDP and STDP synapses. The stimulus is a continuous firing of spikes with $20 \mathrm{~ms}$ of Inter-Spike Interval (ISI). The first $100 \mathrm{~ms}$, the stimulus generated by S11, alone, does not generate any response in the postsynaptic neuron (conditioned stimulus), however, at $100 \mathrm{~ms}$, an unconditioned stimulus (S22) starts firing synchronously and activates the postsynaptic neuron; this concurrent activation strength the synapse with S11 which, after the spikes from S22 have ceased, causes by itself enough depolarization in the postsynaptic neuron to generate an action potential (figures 5 y 6 )

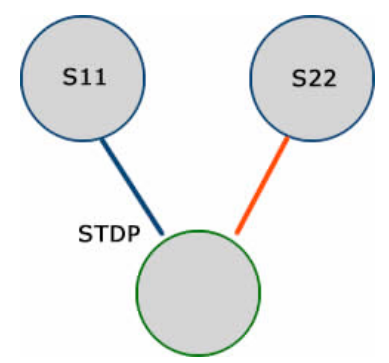

Fig. 4. Configuration of the in-silico experiment to test the STDP potentiation

Effects of STDP in the Pyramidal Neuron (Green)

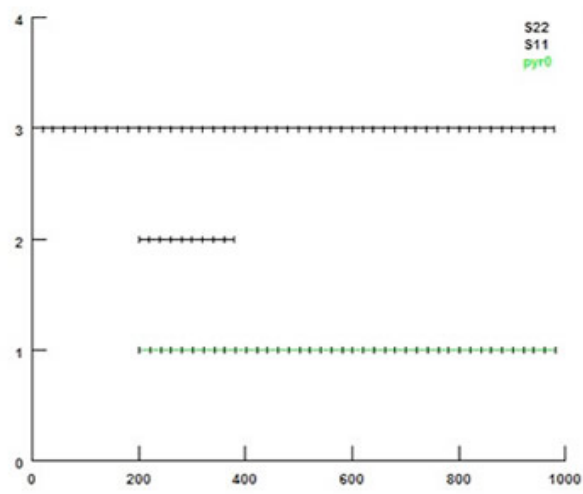

Fig. 5. Effects of STDP in a small network, the synapse with the potentiation mechanism is in the connection between the first neuron from above and the pyramidal neuron (bottom) 


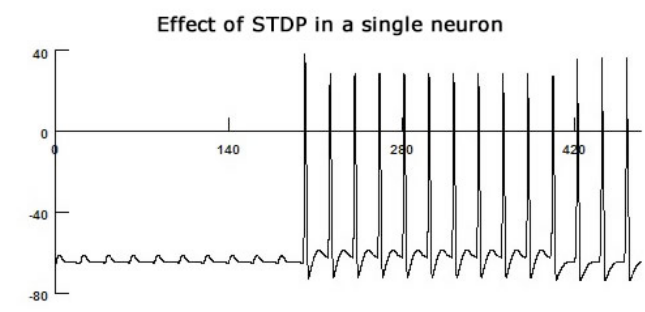

Fig. 6. Action Potentials of the pyramidal network with a STDP synapse showing the potentiation effect

Having the main mechanism of long-term potentiation, the associative networks of Objective and Subjective Value can be proposed, along with the corresponding representation. For the Objective Value, the network shown in figure 7 , associate a stimulus $\mathbf{b}=$ $\left(b_{1}, \ldots, b_{n}\right)$ with a Value $\mathbf{a}=\left(a_{1}, \ldots, a_{n}\right)$, encoded in the sparseness of $\mathbf{a}$ in such a way that low sparseness mean high Objective Value, i.e. The value is directly proportional to the number of neurons active in the population. The firing rate of each individual neuron in the population also can be thought as directly proportional to the value, however, it is not clear still how this could work together with the STDP mechanism.

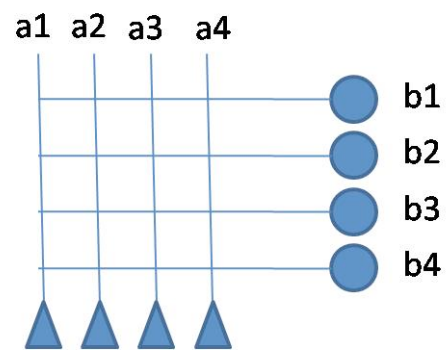

Fig.7. Proposed Objective Value associative network and representation

The Subjective/emotional value, have a similar associative network but in this case, the value is encoded as the proportion of inhibitory or excitatory neurons that are active in a given time, so that, a completely repulsive stimulus activate the entire population of inhibitory neurons and a completely attractive stimulus, cause the same effect in the excitatory pool (figure 8)

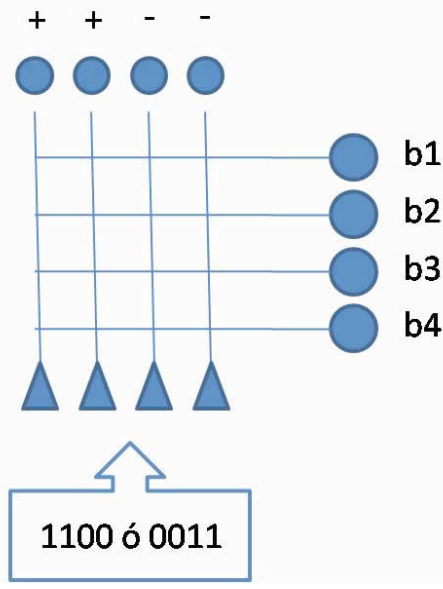

Fig. 8. Proposed Emotional/Subjective Value associative network and representation

\subsection{Competing Values (IGT)}

Now that the appropriate representations of Objective/Emotional Value are set, we can simulate the integration of such values in a hypothetical instance of the IGT in which the subject receive a punishment. In that case, the emotional weight or cue is negative and the real value of the option, even if it appears rationally appealing, is negative, causing low activation in the neurons of the OFC and consequently, turning improbable any behavioral output.

The Network in the OFC/CG that integrate the objective and emotional values, is a recurrent neural network of layers IV or Va of the Cortex, each one with $5 \%$ of probability of connection with any other neuron in the same layer (lateral connections). The Objective and Emotional Values are instrumented with IF Neurons with specific firing rates, each one with noise of 0.2. In figures 9 and 10 , can be seen that, even if the objective value is strong, the activation of the recurrent network is low when inhibited by a negative emotional signal. 


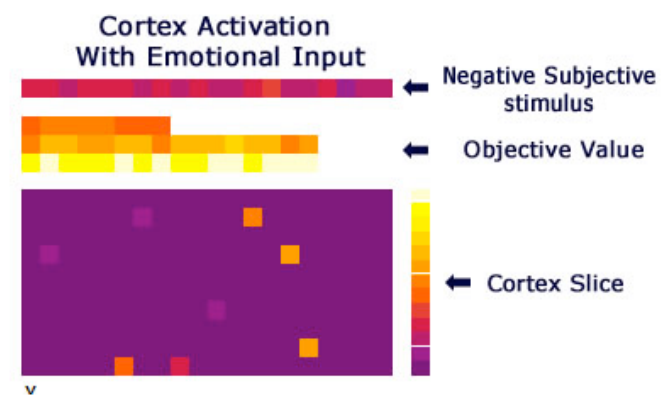

Fig. 9. Activation of the recurrent network of the cortex subject to a negative emotional input or subjective value

\section{Spike Raster of the Inhibited SNN}

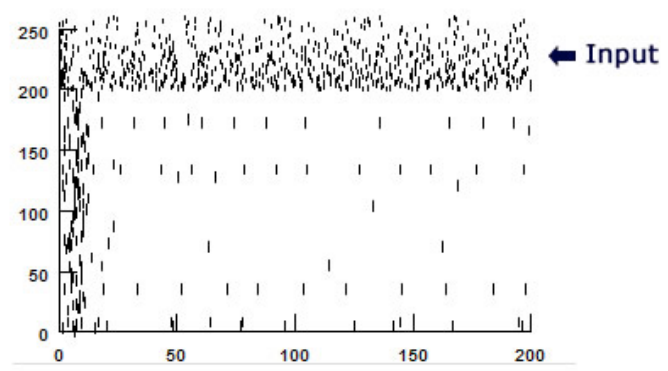

Fig. 10. Neuronal Spikes of the Network inhibited by a negative subjective value

However, when the strength and quantity of the synapses of the projections that convey the objective value increase, they can compete with the emotional inhibition and cause more activation of the recurrent network (figures 11, 12).

Cortex Activation

With Emotional Input and Stronger OV Connections

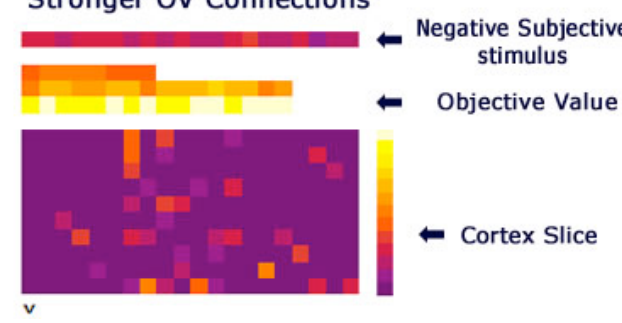

Fig. 11. Activation of the recurrent network of the cortex subject to a negative emotional input or subjective value

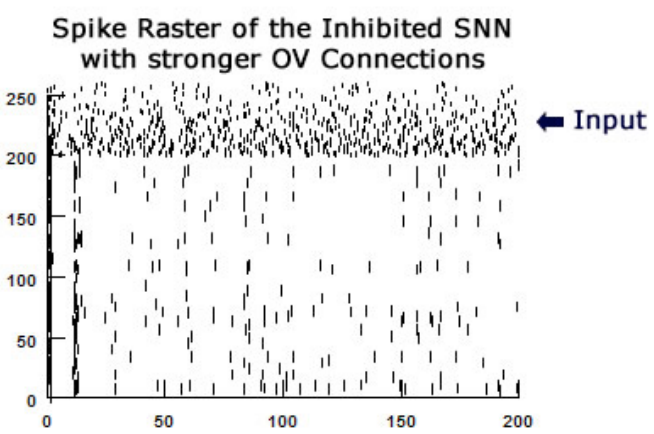

Fig. 12. Neural Spikes of the Network inhibited by a negative subjective value with stronger O.V. connections

On the other hand, deprivation of the emotional signal, for example, by a lesion in the projections that convey such value to the integration network, let the decision making mainly influenced by the reasoned input, the Objective Value, and consequently, the activation of the cortex slice is driven mainly by the population activity related with rational considerations (figures 13 and 14)

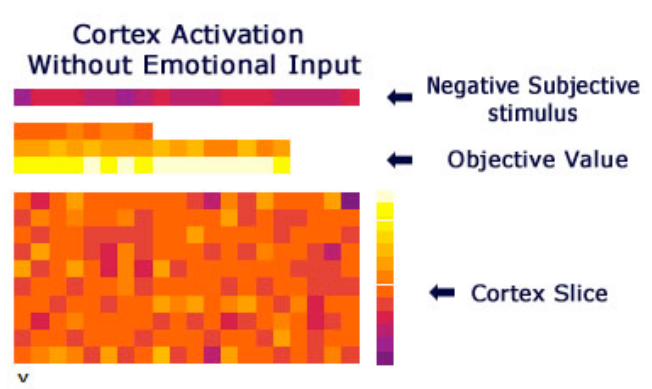

Fig. 13. Activation of the recurrent network of the cortex without negative emotional input

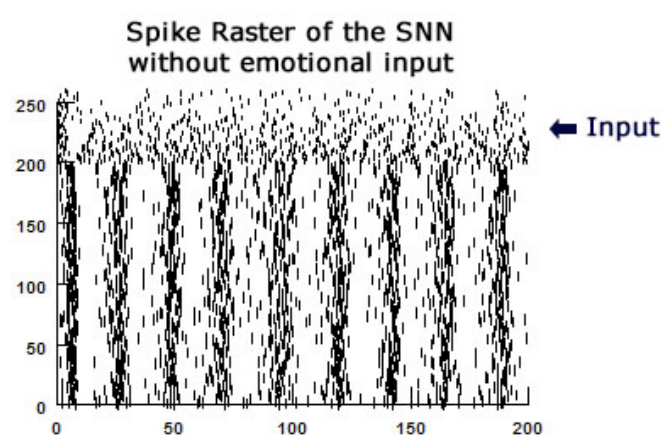

Fig. 14. Neuronal Spikes of the Network without inhibition 


\section{Discussion}

The amount of information available to make a decision can overwhelm us if there is no an emotional bias in each option we consider. The emotional value presented is, obviously not always negative, and can, therefore, favor some options that are rationally undesirable; the other way also applies. Emotions can even disrupt the decision making process when unrelated to the task or options being considered.

The integration and processing of the options in a decision making task, take place in multiple levels and the encoding of different characteristics of the stimuli are still actively studied; hence, this model can only serve us as a test to our understanding about the topic and guide the research in new directions. It is possible that the Somatic Markers, as well as any other alternative explanations of the influence of emotions in decision making could be instances of the same competition illustrated through this model.

\section{Conclusions}

A model of the integration of Objective and Emotional Value was constructed, proposing specific neural representations for each one, based on the population activity. The nature of the specific neural code and the computational aspects of each network within each structure are far from being completely under- stood, however, the simple mechanism presented, can be used to implement the same phenomena in intelligent agents. The simple coding of each value, make it feasible to devise hardware implementations of similar representations based on the sensory and proprioceptive capabilities of such agent.

Future work could revisit the dynamics of the networks involved and take a close look at some network theoretical aspects of the problem and its influence in the cognitive processes under study.

The emotional bias introduced in the model to control the activation of the recurrent network that represent the real subjective value of some option, bears some low level resemblance with the higher level Somatic Marker Hypothesis proposed by Damasio; according to the evidence [Rolls, 2008] it is possible that the neural correlates of such high level considerations could be composed of modules like those studied in this paper and the representations be similar to the proposed ones.

\section{Acknowledgment}

This work was supported by the Universidad Autónoma de Manizales.

\section{References}

[BECHARA, A. et. al. 2005]

[CARNEVALE, N, 2005]

[COLOMBETTI, G, 2008

[DAMASIO, A. et al. 1991]

[DAMASIO, A. 1994]

[DAMASIO, H. et al. 1994]
BECHARA, Antonie., DAMASIO, Antonio. The Somatic Marker Hypothesis: A neural theory of economic decision.

Games and Economic Behavior, 52 (2005) 336-372.

CARNEVALE, Nicholas T. The Neuron Book.

Cambridge University Press. 2005.

COLOMBETTI, Giovanna. The Somatic Marker Hypoteses, and what the

Iowa Gambling Task does and does not show.

British Journal for the Philosophy of Science. 59 (2005) 51-71.

DAMASIO, Antonio., TRANEL D., DAMASIO, Hanna. Somatic Markers

and the guidance of behaviour: Theory and preliminary teesting.

Jenkins, J. M, Oatley, K., Stein, N. L. Human Emotions: a reader, Willey

- Blackwell. 1998.

DAMASIO, Antonio. Descartes' Error: Emotion, Reason and the Human Brain.

Putnam Publishing, 1994.

DAMASIO, Hanna., GRABOWSKY, Thomas., FRANK, Randall., GALABURDA,

Albert M., DAMASIO., Antonio. The return of Phineas Gage: Clues About the 
[DAYAN, P. et al. 2001]

[DUNN, B. et al. 2006]

[FELLOUS, J. et al. 2005]

[FELLOUS, J. 2007]

[GLIMCHER, P. et. al. 2009]

[MACMILLIAN, M. 2009]

[LEDOUX, J. 2000]

[LITT, A. et. al. 2008]

[PLATT, M.et.al.2009]

[ROLLS, E. et.al. 2008]

[THAGARD, P. 2008]
Brain form the Skull of a Famous Patient.

Science, 264(5162) (1994) 1102-1105

DAYAN, Peter., ABBOTT, L. F. Theoretical Neuroscience: Computational and Mathematical Models of Neural Systems.

MIT Press, 2001.

DUNN, Barnaby D., DALGLEISH, Tim., LAWRENCE, Andrew D. The

somatic marker hypothesis: A critical evaluation.

Neuroscience and Biobehavioral Reviews. 30 (2006) 239-271.

FELLOUS, Jean M., ARBIB, Michael A. Who Needs Emotions? The Brain

Meets the Robot.

Oxford University Press, 2005.

FELLOUS, Jean M. Models of Emotion.

Scholarpedia, 2007.

GLIMCHER, Paul., CAMERER, Colin., FEHR, Ernst., POLDRACK, Russell A. (eds). Neuroeconomics: Decision Making and the Brain.

Academic Press, 2009.

MACMILLIAN, Malcolm. The Phineas Gage Information Page [En línea]. http://www.deakin.edu.au/hmnbs/psychology/gagepage/.

LEDOUX, Joseph E. Emotion Circuits in the Brain.

Annu. Rev. Neurosci, 23 (2000) 155-184.

LITT, Abninder., ELIASMITH, Chris., THAGARD, Paul. Neural Affective

Decision Theory: Choices, Brains, and Emotions.

Neural Affective Decision Theory: Choices, Brains, and Emotions, 9 (2008) 252273.

PLATT, Michael., SCHIOPPA-PADOA, Camilo. Neuronal Representation of Value. Glimcher et. al. (eds.), Neuroeconomics: Decision Making and the Brain. Academic Press 2009.

ROLLS, Edmund T. Memory, Attention and Decision-Making.

Oxford University Press. Academic Press 2008.

THAGARD, Paul. Spiking Phineas Gage: A Neurocomputational Theory

of Cognitive-Affective Integration in Decision Making.

Thagard, P. Hot Thoughts. MIT Press 2006. 